\title{
Experimental observation of parametric effects near period doubling in a loss-modulated $\mathrm{CO}_{2}$ laser
}

\author{
V. N. Chizhevsky* \\ B. I. Stepanov Institute of Physics, Belarus Academy of Sciences, 220072 Minsk, Republic of Belarus \\ Ramón Corbalán \\ Departament de Fisica, Universitat Autònoma de Barcelona, E-08193 Bellaterra, Barcelona, Spain
}

(Received 17 October 1995 )

\begin{abstract}
A number of parametric effects, such as suppression of period doubling, shift of the bifurcation point, scaling law relating the shift and the perturbation amplitude, influence of the detuning on the suppression, reaching of the maximum gain between the original and shifted bifurcation points, and scaling law for idler power are experimentally observed near period doubling bifurcation in a loss-driven $\mathrm{CO}_{2}$ laser that is subjected to periodic loss perturbations at a frequency that is close to a parametric resonance of the unperturbed system.
\end{abstract}

PACS number(s): 42.55.Lt, 05.45.+b, 0.5.70.Jk, 42.50.Lc

Over the last years considerable attention has been paid to the effect of periodic perturbations on the dynamics of nonlinear systems near simple bifurcation points. Depending on the type of bifurcation (supercritical or subcritical), the periodic perturbations can produce stabilizing or destabilizing shifts of bifurcation points in dynamical system [1-5]. The stabilizing effect of periodic perturbations on the dynamics of nonautonomous systems as well as a great number of related nonlinear parametric effects for the case of a generic supercritical period-doubling bifurcation has been predicted in Ref. [1] from the analysis of normal forms describing essential dynamics on low-dimensional manifolds. A similar result concerning the stabilization and another set of predictions has been obtained in Ref. [3] from an analysis, linear in perturbations, of a simple one-dimensional iterative model describing the dynamics in the Poincare map. Experimentally, the suppression of instabilities (i.e., the stabilizing shift of bifurcation points) has been observed in a number of systems such as analog circuits [1], Josephson junctions [2,4], and magnetostrictive ribbons [5]. The destabilizing effect of periodic perturbations near a subcritical bifurcation point has been experimentally and theoretically shown in Ref. [6]. More recently, Vohra, Fabini, and Bucholtz reported experimental results which demonstrate that the onset of chaos in a nonlinear system can be either suppressed or induced by near-resonant perturbations [7].

In this paper we report the results of an experimental observation of a variety of parametric effects near perioddoubling bifurcation (PDB) in a loss-modulated $\mathrm{CO}_{2}$ laser which is subjected to additional periodic loss perturbations. The modulated $\mathrm{CO}_{2}$ laser is known to display, with a large signal-to-noise ratio, a rich phenomenology of nonlinear effects, and depending on experimental conditions may exhibit both types of bifurcations [8]. Here we consider the case of a

\footnotetext{
*Present address: Departament de Fisica, Universitat Autònoma de Barcelona, E-08193 Bellaterra, Barcelona, Spain. Fax: 7-0172-393131. Electronic address: ifanbe\%bas03.basnet. minsk.by@demos.su

${ }^{\dagger}$ Fax: 34-3-5812155. Electronic address: ifop0@cc.uab.es
}

supercritical period-doubling bifurcation (PDB). In contrast with the experiments in Ref. [5], we explore the influence of periodic perturbations with a large detuning from the subharmonic frequency. We have observed a number of effects predicted in Ref. [1]: the suppression of period doubling and the shift of the bifurcation point with increasing amplitude of the loss perturbation; a scaling law relating the shift of the bifurcation point and the perturbation amplitude; and a parametric amplification at the perturbation frequency as a function of the perturbation amplitude as well as the discontinuity in the laser response at a critical perturbation amplitude. These effects were observed earlier in the strain dynamics of magnetostrictive ribbons [5] in the case of near-resonant perturbations. In our work, owing to the large detuning used, we could also experimentally observe the reaching of maximum laser sensitivity to the perturbation between the original (unperturbed system) and shifted bifurcation points and the influence of the detuning on the "sharpness" of perioddoubling suppression. According to the discussion in Ref. [1], these last two observations are only possible in the socalled "dynamic regime," when the detuning is large enough to be significant. Precisely in this context we say that we used large detunings. A more precise definition of the ranges of detuning which characterize the large and small detuning regions would require knowing the normal form which governs the dynamics of the perturbed bifurcation, but this is left for future work.

For small perturbation signals we have experimentally found a scaling law relating the idler-to-signal amplitude ratio and the bifurcation parameter that shows the idler power rise with increasing of the control parameter. This scaling law is in a good agreement with the theoretical prediction of Swensmark and Wiesenfeld [9].

The experimental setup is essentially the same as in our previous studies [10]. The investigations were performed with a cw single-mode frequency-stabilized $\mathrm{CO}_{2}$ laser operating on the $10 P(22)$ line. The resonator losses were modulated via two acousto-optic modulators placed inside the cavity. On one of them we applied a sinusoidal voltage $V_{d}(t)=V_{d} \cos \left(2 \pi f_{d} t\right)$ which drives the laser in the vicinity of the $T-2 T$ PDB, where $T=1 / f_{d}$. This voltage is hereafter 
called the "driving" voltage. On the other modulator we applied a sine wave voltage $V_{s}(t)=V_{s} \cos \left(2 \pi f_{s} t\right)$, called the "signal," to probe the laser response to this loss perturbation. The signal frequency is detuned by $\delta$ from $f_{d} / 2: f_{s}=f_{d} / 2+\delta$. The homemade acousto-optic modulators were adjusted in such a way that one of them had an acoustic resonance at the driving frequency $f_{d}$, and the other modulator at the signal frequency $f_{s}$. We checked on both modulators that the loss changes induced by the driving and signal voltages had sine wave character as well as linear dependence on the amplitude voltage $V_{d}$ and $V_{s}$ over the range of values used in the present experiments. In all our experiments the loss changes induced by the signal $V_{s}(t)$ did not exceed $20 \%$ of those induced by the driving voltage $V_{d}(t)$, whose amplitude $V_{d}$ reached values of the order of $7 \mathrm{~V}$ at the PDB point. The laser output intensity was measured by a $\mathrm{Hg}$-Cd-Te detector with a time resolution of $50 \mathrm{~ns}$ and a digital oscilloscope coupled to a PC/AT486. For numerical analyses the signals were digitized with sampling frequencies up to $20 \mathrm{MHz}$, with eight-bit resolution and 4096 samples per recording.

Laser parameters such as frequency $f_{r}$ and decay constant $\gamma$ of relaxation-oscillation were measured from the laser response to short-lived optically induced loss perturbations. This technique was successfully used earlier for investigating transient dynamics in $\mathrm{CO}_{2}$ lasers [11-13]. Laser parameters such as the cavity length and the discharge current were chosen so that the driving frequency $f_{d}$ was twice the relaxationoscillation frequency of the $\mathrm{CO}_{2}$ laser. Although this is not necessary, and in fact cases with both $f_{d}<f_{r}$ [10] and $2 f_{r}>f_{d}>f_{r}$ [14] have been investigated previously, the choosing of the driving frequency $f_{d}=2 f_{r}$ in the present investigations was determined by two reasons: (i) in this case we have exact parametric resonance, and (ii) in this case the modulated output of the $\mathrm{CO}_{2}$ laser is significantly more stable than for the case $f_{d}<f_{r}$. In all the experiments reported here $f_{r}=50 \mathrm{kHz}, \gamma=100 \mathrm{kHz}$, and $f_{d}=100 \mathrm{kHz}$. The driving amplitude $V_{d}$ is the bifurcation parameter. The detuning $\delta$ was varied in the range going from $0.5 \mathrm{kHz}(0.01$, in units of $\left.f_{d} / 2\right)$ to $5 \mathrm{kHz}\left(0.1 f_{d} / 2\right)$. For comparison note that the detunings used in Ref. [5] did not exceed $10^{-4} f_{d} / 2$.

The results graphed in Fig. 1 show the response of the loss-modulated $\mathrm{CO}_{2}$ laser above the $T-2 T$ PDB to loss perturbations induced by $V_{s}(t)$. We measured the power Fourier transform $I(f)$ of the laser intensity $I(t)$ at frequency $f_{d} / 2$ of the original period doubling, at signal frequency $f_{s}$, and at idler frequency $f_{i}=f_{d} / 2-\delta$. For normalization purposes we also measured $I_{0}$, the Fourier component of the laser intensity at the signal frequency in the absence of the driving voltage. Parts (a), (b), and (c) of Fig. 1 represent $I\left(f_{d} / 2\right)$, $I\left(f_{s}\right)$, and the idler-to-signal amplitude ratio $R=I\left(f_{i}\right) / I\left(f_{s}\right)$, respectively, as a function of the perturbation amplitude $V_{s}$. Curves $1-3$ correspond to different detunings. As the perturbation amplitude increases, the laser intensity spectrum shows the appearance of peaks evenly spaced around $f_{d} / 2$ by the interval $\delta$. When the amplitude $V_{s}$ reaches a critical value $\left(V_{s} \approx 0.4 \mathrm{~V}\right.$ for $\delta=0.01$, curve $1: V_{s} \approx 0.9 \mathrm{~V}$ for $\delta=0.04$, curve 2; and $V_{s} \approx 2.1 \mathrm{~V}$ for $\delta=0.08$, curve 3), used to rescale the horizontal axis in Fig. 1, the signal at $f_{d} / 2$ [Fig. 1(a)] and all even extra peaks vanish, but odd ones increase, showing switchlike behavior [Fig. 1(b)]. Above the critical point only odd components spaced by an interval of $2 \delta$ remain in the
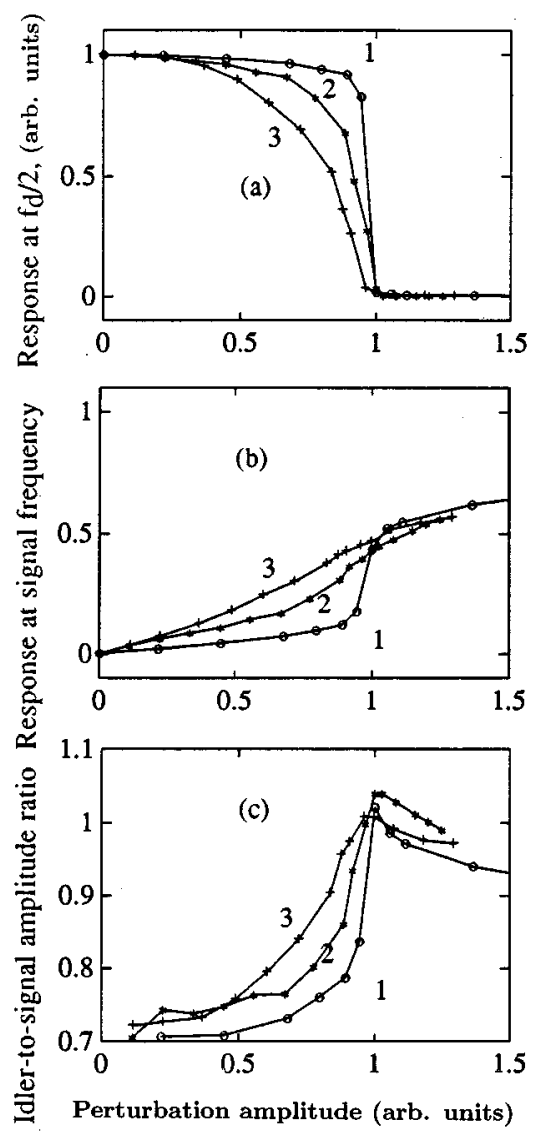

FIG. 1. Laser response above PDB as a function of perturbation amplitude for different values of the detuning $\delta$ : (1) $\delta=0.01$, (2) $\delta=0.04$, and (3) $\delta=0.08$. (a) The laser response at frequency $f_{d} / 2$. (b) The laser response at signal frequency $f_{d} / 2+\delta$. (c) The idler-tosignal amplitude ratio $R$. The perturbation amplitude is normalized to a critical value, given in the text, different for each detuning.

laser intensity spectrum, and since the original component at $f_{d} / 2$ is missing, one speaks of suppression of PDB. A comparison of the results graphed in the three curves of Fig. 1(a) (with a different horizontal rescaling for each curve) shows that by increasing the detuning from 0.01 (curve 1) to 0.08 (curve 3) the character of the period-doubling suppression passes from switchlike at small detuning up to continuous at large detuning. Such a behavior with increasing detuning is typical of all other spectral components in the laser response [Fig. 1(b)]. A similar effect is also observed in the idler-tosignal amplitude ratio which is plotted in Fig. 1(c), and also demonstrates a switchlike behavior at small detuning and a smooth one at large $\delta$. When the response at $f_{d} / 2$ drops to zero, $R$ reaches its maximum value very close to 1 . This means that in this point the system reaches another bifurcation point [9]. The point where the ratio $R$ has a maximum value can be used as a criterion in the experimental determination of the bifurcation point in dynamical systems in the presence of a perturbation signal and noise. We stress this fact because, as was pointed out in Ref. [3], it is in general impossible to determine the actual bifurcation point in the presence of perturbations by an analysis of the power spectrum.

To further document the influence of the detuning on the 

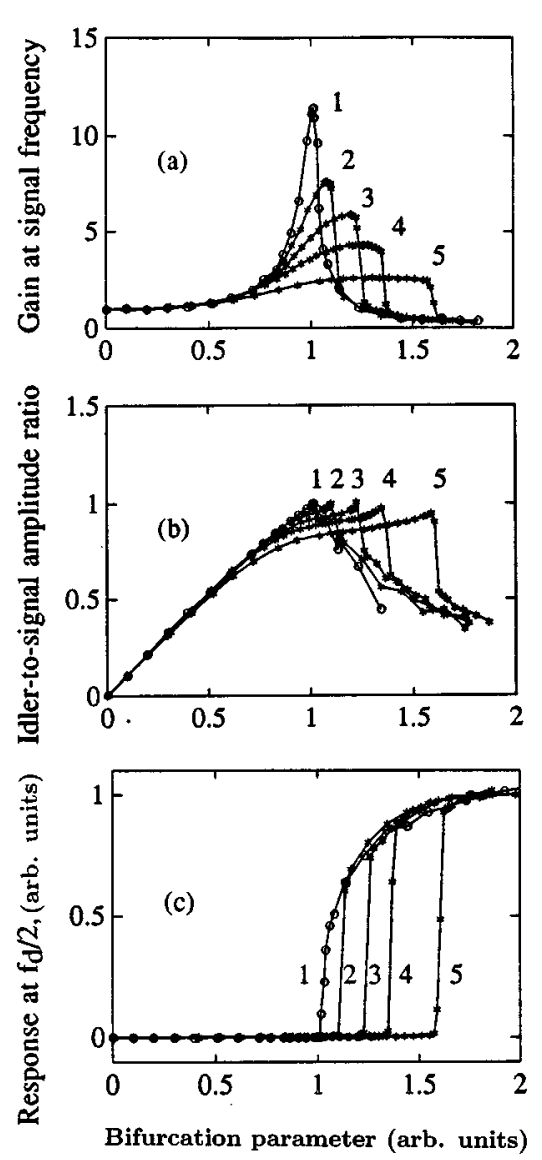

FIG. 2. Laser response as a function of the bifurcation parameter for different values of the perturbation amplitude $V_{s}$ (arb. units). For curves $1-5, V_{s}=1,3.8,6.3,10.4$, and 20, respectively. (a) Parametric gain at signal frequency. (b) The idler-to-signal amplitude ratio. (c) The laser response at $f_{d} / 2$ showing the shift of the bifurcation point. The bifurcation parameter is normalized to the value at the bifurcation point with $V_{s}=0$. Here $\delta=0.01$.

rapidity with which the amplitude of period doubling (i.e., the laser response at $f_{d} / 2$ ) changes near PDB, we kept the perturbation amplitude and the detuning fixed and measured the interval $\Delta V_{d}$ of control parameter in which the amplitude of period doubling $I\left(f_{d} / 2\right)$ changes from the level 0.8 of its initial value (without perturbation) to 0 . We obtained almost a linear dependence of $\Delta V_{d}$ on $\delta: \Delta V_{d} \propto \delta^{0.9}$.

The results in Fig. 2 correspond to the laser behavior when the signal amplitude and detuning are fixed and the bifurcation parameter $V_{d}$ is varied. The value of $V_{d}=1$ corresponds to the original bifurcation point of the unperturbed $\mathrm{CO}_{2}$ laser (i.e., with $V_{s}=0$ ). Various curves in Figs. 2(a)2(c) correspond to different values of the signal amplitude. Figure 2(a) shows the gain $G=I\left(f_{s}\right) / I_{0}$ for parametric amplification at the signal frequency. It should be noted that various aspects of the phenomena of signal amplification and classical squeezing or deamplification were investigated earlier in loss-modulated $\mathrm{CO}_{2}$ lasers $[10-12,14,15]$. Here we provide additional experimental information on this problem. When the perturbation amplitude is very small there is not any peculiarity in the dependence of the gain on the bifurcation parameter [Fig. 2(a), curve 1]. This case corresponds to the linear regime of small signal amplification, and it has been intensively studied both theoretically $[9,16]$ and experimentally in diverse dynamical systems $[10-12,14,15,17-19]$. When the perturbing signal is large enough so that nonlinearities play a significant role in the laser response, we observe a discontinuity in the parametric gain $G$ [Fig. 2(a), curves 2-5]. A similar behavior is also observed in the idler-to-signal amplitude ratio [Fig. 2(b)]. Above the shifted bifurcation point (i.e., above the value of $V_{d}$ at which $R$ reaches its maximum value) we have strong signal deamplification up to the next $2 T-4 T$ bifurcation point. Similar results were observed earlier by us in the case of small detuning in phase-averaged measurements [10]. These results mean that in the frequency band going from $f_{d} / 2-\delta$ to $f_{d} / 2+\delta$ the system becomes significantly less sensitive to perturbations (not only periodic). For the case represented in Fig. 2(a) the gain $G$ decreases by a factor 5 in going from the asymptotic left-hand side value to the asymptotic right-hand side one. This effect may be very useful from the point of view of some practical applications, because it allows us to obtain a source of IR emission with effectively reduced noise in a small frequency band near $f_{d} / 2$.

For the case of large enough detunings, it has been predicted in Refs. [1] and [3] that the maximum gain at signal frequency always occurs between the original and shifted bifurcation points. One can see the experimental confirmation of these predictions by a comparison of Figs. 2(a) and 2(b) which show that the values of the bifurcation parameter where $G$ and $R$ reach their maxima are different. This effect is especially conspicuous for large signal amplitudes, but it also exists for small signal. This difference means that the maximum of the gain $G$ lies between the original and shifted bifurcation points, because the criterion for the appearance of $\mathrm{PDB}$, as mentioned above, is the reaching of the maximum of the idler-to-signal amplitude ratio.

From the results in Fig. 2(b) one can check experimentally an important result which has been theoretically predicted in Ref. [9]: a scaling law relating the ratio $R$ of idlerto-signal power amplitudes and the bifurcation parameter $V_{d}$. In this reference it has been shown that for nonlinear dynamical systems near PDB the ratio $R$ is a function of the dissipation $k=\gamma T$, the detuning $\delta$, and the control parameter $V_{d}$, which are related by the simple scaling expression $R=V_{d} /\left[1+(2 \delta / k)^{2}\right]^{1 / 2}$ (in our notations). This expression was obtained from a set of model equations for a Poincare return map for the case of small dissipation and detuning in the system. In our experimental conditions $\delta=0.01$ and $k=\gamma T=1$. Although the quantity $k$ is not small, the curve 1 in Fig. 2(b) shows that the proportional dependence of $R$ on $V_{d}$ is kept in a wide range of the bifurcation parameter going from 0 up to 1 . In this interval the slope of curve 1 is 0.96 \pm 0.02 , that is very close to 1 , the value predicted by the above formula. An increase of the signal amplitude [curves $2-5$ in Fig. 2(b)] leads to a decrease of the range of bifurcation parameter where there is a proportional dependence of $R$ on $V_{d}$, and also to a decrease of the maximum value of $R$.

The shift of the bifurcation point is clearly seen in Fig. 2(c), which represents $I /\left(f_{d} / 2\right)$ as a function of the bifurcation parameter for different values of perturbation amplitude. Curve 1 corresponds to a signal of very small amplitude, and almost coincides with the rise of this Fourier component of the laser intensity after PDB of the driven laser. When the amplitude of the perturbation signal is large enough, we ob- 

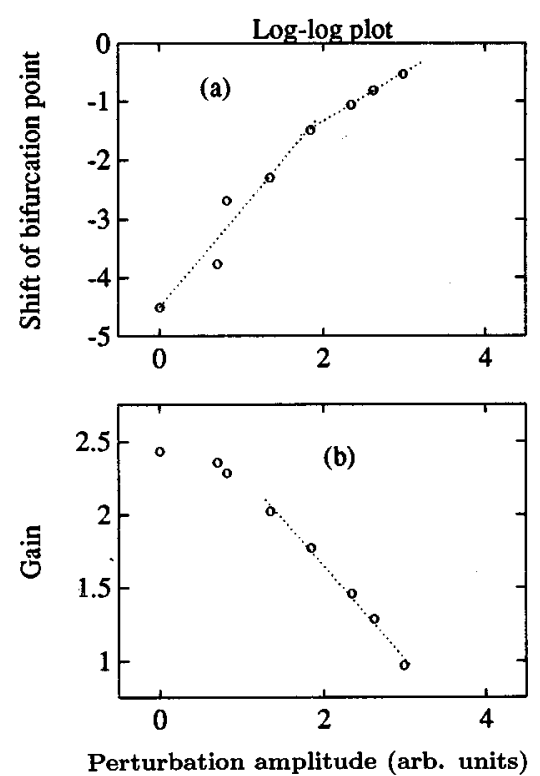

FIG. 3. (a) Shift of the bifurcation point, and (b) maximum signal gain as a function of the perturbation amplitude. All parameters are as in Fig. 2. Scales are $\log _{e}$.

serve a shift of the bifurcation point to a value which is greater than the original one, as well as a discontinuity in the laser response at the frequency $f_{d} / 2$. The dependence of this shift and of the maximum gain on the perturbation amplitude are shown in the log-log scale in Figs. 3(a) and 3(b), respectively. In our experiments the perturbation amplitude, measured in terms of $I_{0}$, was changed approximately by 25 times from small to large enough signal. In Fig. 3(a) one sees two regions in which the curve has a different slope. The first region corresponds to small perturbations, and the slope is $\alpha=1.65$, that is less than the value of 2 predicted in Ref. [3] by a linear analysis for small signals. In the second region we have $\alpha=0.84$, that is also different from the value $\frac{2}{3}$ predicted in Ref. [1] and experimentally confirmed in Ref. [5]. Obviously, this difference is connected with the relatively large detuning we had in our experiments, while the experiments of Ref. [5] were performed at very small detuning $\delta$.

From the data in Fig. 3(b) one obtains another scaling law relating the maximum gain $G$ and the perturbation amplitude $I_{0}$. These quantities are related by the following relationship: $G \propto I_{0}^{-\alpha}$, where $\alpha=0.64$ that is very close to the value of $\frac{2}{3}$ which was obtained by us for very small detuning in phaseaveraged measurements [10], and also predicted for a different $\mathrm{CO}_{2}$ laser [14]. This means that, contrary to the shift, the exponent in the scaling law relating $G$ and $I_{0}$ does not practically depend on the detuning $\delta$.

In conclusion, a number of parametric effects near PDB such as the suppression of PDB, the shift of the bifurcation point, the influence of detuning on the suppression, the reaching of the maximum parametric signal gain between original and shifted bifurcation points, and various scaling laws were observed in the dynamics of a periodically perturbed loss-driven $\mathrm{CO}_{2}$ laser.

V.N.C. acknowledges partial support of this work by International Science Foundation (Grant No. MX3000) and the DGICYT (Spain) for a grant (SB94-A26). R.C. acknowledges financial support from the DGICYT (Contract No. PB92-0600-01).
[1] P. Bryant and K. Wiesenfeld, Phys. Rev. A 33, 2525 (1986).

[2] H. Swensmark, J. B. Hansen, and N. F. Pedersen, Phys. Rev. A 35, 1457 (1987).

[3] H. Swensmark and M. R. Samuelsen, Phys. Rev. B 41, 4181 (1990).

[4] G. F. Ericsen and J. B. Hansen, Phys. Rev. B 41, 4189 (1990).

[5] S. T. Vohra, F. Bucholtz, K. P. Koo, and D. M. Dagenais, Phys. Rev. Lett. 66212 (1991); ibid. 66, 2843 (1991).

[6] S. T. Vohra, L. Fabiny, and K. Wiesenfeld, Phys. Rev. Lett. 72, 1333 (1994).

[7] S. T. Vohra, L. Fabiny, and F. Bucholtz, Phys. Rev. Lett. 75, 65 (1995).

[8] J. R. Tredicce, F. T. Arecchi, G. P. Puccioni, A. Poggi, and W. Gadomski, Phys. Rev. A 34, 2073 (1986); D. Dangoisse, P. Glorieux, and D. Hennequin, Phys. Rev. A 36, 4775 (1987); R. Meucci, A. Poggi, F. T. Arecchi, and J. R. Tredicce, Opt. Commun. 65, 151 (1988); C. O. Weiss and R. Vilaseca, Dynamics of Lasers (VCH, Veinheim, 1991).

[9] H. Swensmark and K. Wiesenfeld, Phys. Rev. A 46, 787 (1992).

[10] R. Corbalan, J. Cortit, V. N. Chizhevsky, and A. N. Pisarchik,
Infrared Phys. Technol. 36, 71 (1994); R. Corbalan, J. Cortit, A. N. Pisarchik, V. N. Chizhevsky, and R. Vilaseca, Phys. Rev. A 51, 663 (1995).

[11] A. M. Samson, S. I. Turovets, V. N. Chizhevskii, and V. V. Churakov, Zh. Eksp. Teor. Fiz. 101, 1177 (1992) [Sov. Phys. JETP 74, 628 (1992)].

[12] V. N. Chizhevsky and S. I. Turovets, Opt. Commun. 102, 175 (1993).

[13] V. N. Chizhevsky and P. Glorieux, Phys. Rev. E 51, R2701 (1995).

[14] P. Glorieux, C. Lepers, R. Corbalan, J. Cortit, and A. N. Pisarchik, Opt. Commun. 118, 309 (1995).

[15] C. Lepers, J. Legrand, and P. Gloriex, Phys. Lett. A 161, 493 (1992).

[16] K. Wiesenfeld and B. McNamara, Phys. Rev. Lett. 55, 13 (1986); Phys. Rev. A 33, 629 (1986).

[17] B. Derighetti, M. Ravani, R. Stoop, P. F. Meier, E. Brun, and R. Badii, Phys. Rev. Lett. 55, 1746 (1985).

[18] M. Bocko and J. Battiato, Phys. Rev. Lett. 60, 1763 (1988).

[19] S. Martin and W. Martinsen, Phys. Rev. A 34, 4523 (1986). 